15 ASSESSMENT OF ADHERENCE TO INTERNATIONAL ANTIBIOTIC PRESCRIPTION GUIDELINES IN DENTAL CLINICS IN PRIMARY HEALTHCARE CENTERS IN NATIONAL GUARD, WESTERN REGION, SAUDI ARABIA

${ }^{1}$ Ayshah Melaibari, ${ }^{2}$ Nada Naaman, ${ }^{3}$ Hafsah Al-Ali, ${ }^{4}$ Reem Baghareeb, ${ }^{5}$ Nada AlMutairi, ${ }^{6}$ Bashair Aldosari, ${ }^{7}$ Saad Al Ghamdi, ${ }^{7}$ Taghreed Aldosary. ${ }^{1}$ College of Dentistry, Princess Nourah Bint Abdulrahman University, Riyadh, SA; ${ }^{2}$ College of Medicine, King Saud Bin Abdulaziz University for Health Sciences, Jeddah, $S A ;{ }^{3}$ College of Dentistry, King Saud University, Riyadh, SA; ${ }^{4}$ Quality Dental Clinic, Makkah, SA; ${ }^{5}$ College of Dentistry, AlFarabi Private College, Jeddah, $S A ;{ }^{6}$ College of Medicine, Ibn Sina National College for Medical Studies, Jeddah, SA; ${ }^{7}$ Community \& Preventive Medicine, National Guard Health Affairs, Jeddah, SA

\subsection{6/bmjoq-2019-PSF.15}

Background Antibiotics have played a major role in the treatment of infectious diseases since 1928; however, the overuse of antibiotics has contributed to the development of antibioticresistant pathogens. According to the Centers for Disease Control and Prevention (CDC), at least 30\% of prescribed antibiotics are unnecessary in the USA. Following the CDC checklist, practitioners should avoid non-evidence-based historical prescriptions and ensure that prescriptions are not written on the basis of patient expectations, convenience, or pressure from colleagues. In certain cases, such as when a patient present with extra-oral swelling, trismus, or the need for a surgical intervention, an appropriate regimen of antibiotics is indicated; otherwise, systemic antibiotics should not be the routine treatment of choice. This study aims to determine and assess adherence to CDC guidelines for antibiotic prescriptions in dental clinics at National Guard Primary Healthcare Centers, Western Region, Saudi Arabia.

Methods The study was carried out at National Guard Primary Healthcare Centers in Western Region, Saudi Arabia. Records of all patients with antibiotic prescriptions during their dental visits from January 2017 to June 2018 were sampled through simple random sampling; medically compromised patients were excluded. Dentists' adherence to the guidelines was assessed. MS Excel was used for data entry, and SPSS version 23 was used to analyze the data, using the chi-squared test for significance.

Results A total of 385 clinical records were reviewed, of which $30 \%$ of the antibiotics were ordered without proper documentation of the diagnosis. Only $11.2 \%$ of the prescriptions adhered to the guidelines, while the other $88.8 \%$ did not follow the guidelines, including improper documentation. There was a significant difference between the prescriptions that did and did not follow the guidelines. On the other hand, the relation between the dentist's level of education and adherence to the antibiotic prescription guidelines was not significant.

Conclusion There is inadequate adherence to the international guidelines among dental practitioners in dental clinics in primary healthcare centers in the National Guard. This irrational antibiotic prescribing accounts for the risk of antibiotic resistance, so there is a need to address antibiotic prescribing practices by increasing awareness among dental practitioners in these clinics.

16 PATIENT-BASED RETROSPECTIVE DOSE AUDIT FOR COMMON RADIOGRAPHIC EXAMINATIONS

Bushra Alghamdi, Rawan Hanain, Marwah Alshatri, Haneen Alshehri, Shaza Alsharif, Khalid Alshamrani. Radiological sin, Saud bin Abdulaziz University for Health Sciences
Background Retrospective dosimetric auditing is a useful investigational approach for continuous quality assurance in diagnostic radiography. It may serve as a baseline reference for future research in which both dose and image quality are examined. In radiation protection, comparing diagnostic reference levels (DRLs) with patient doses observed in clinical practice enables the optimization process (i.e., providing a clinically acceptable image quality and keeping the dose as low as reasonably achievable). The purpose of this study was to audit radiation doses of adult patients who underwent common diagnostic $\mathrm{x}$-ray examinations and compare dose area product (DAP) values with the established international DRLs (IDRLs).

Methods Retrospective cross-sectional records of 193 patients who underwent 387 radiographic examinations during October through December 2018 were obtained. Patient-related and DAP data were recorded for the six most common examinations with two digital systems of the same manufacturer at King Abdulaziz Medical City in Jeddah (KAMC-JD). The mean and 75 th percentile of DAPs was established and compared with IDRLs. The percentage mean and 75th percentile DAP differences were used to describe changes between KAMC-JD values and IDRLs.

Results Relative to chest and cervical spine x-rays, wider dispersion and increased variability in DAP values were observed and evidenced by a larger standard deviation for lumbar spine (AP $\sigma=170.29$; lateral $\sigma=409.92$ ), thoracic spine (AP $\sigma=43.22$; lateral $\sigma=440.44)$, abdomen $(\sigma=131.62)$, and pelvis $(\sigma=254.59)$. Overall, the DAP 75 th percentiles for KAMC-JD were below IDRLs for chest, cervical spine, abdomen, and pelvis. Lateral lumber, and AP and lateral thoracic spine DAP 75th percentile exceeded all IDRLs by up to $42.56 \%, 1.68 \%$, and $512.17 \%$, respectively. When the type of detector is considered, KAMCJD mean DAPs exceeded the UK 2012 DRLs for the following examinations: AP and lumbar spine $(42.83 \%, 110.8 \%)$, AP pelvis $(8.06 \%)$, AP and lateral cervical $(62.86 \%, 10.71 \%)$, and $\mathrm{AP}$ and lateral thoracic $(36.27 \%, 355.78 \%)$.

Conclusion KAMC-JD DAP data were below the international recommendations except for lumbar spine, which exceeded the recommendations. Reassessment after optimizing the lumbar spine DRL is recommended. This study highlights the need for developing local DRLs in Saudi Arabia at the institutional, regional, and national level.

\section{DO PATIENTS UNDERSTAND WHAT THEY CONSENT FOR? ANALYSIS OF DISCORDANCE IN PATIENT- PHYSICIAN UNDERSTANDING OF THE CHEMOTHERAPY PLAN}

Hani Mesfer Almalki, Ahmad Almohanad Absi, Abdurrahman Abdullah Alghamdi, Mohammed Abdullah Alsalmi, Mohammed Anwar Khan. College of Medicine, King Saud bin Abdulaziz University for Health Sciences

\subsection{6/bmjoq-2019-PSF.17}

Background The journey towards better patient safety starts with effective patient-physician communication. This is true for cancer patients, in whom treatments are complex and patients' understanding might be compromised. Multiple factors can result in discordance in patient-physician agreement including patients' and physicians' personal factors, complexity of the disease, and the setting of care. The present work investigates the discordance between patients and their doctors in understanding the chemotherapy plan in Princess Norah Oncology Center, Jeddah. 